\title{
Aprender a Evaluar mediante Juegos de Simulación en Educación Superior: Percepciones y Posibilidades de Transferencia para los Estudiantes
}

\author{
Learn to Assess through Simulation Games in Higher \\ Education: Perceptions and Transfer Possibilities for Students
}

\author{
Miguel Ángel Gómez-Ruiz * \\ María Soledad Ibarra-Sáiz \\ Gregorio Rodríguez-Gómez \\ Universidad de Cádiz, España
}

\begin{abstract}
La utilización creciente en Educación Superior de videojuegos serios, es decir, aquellos con intencionalidad educativa, se justifica por el enriquecimiento experiencial de los estudiantes. Dentro de estos, en este trabajo nos centramos en los juegos de simulación, recreaciones de la realidad que permiten vivenciar desde la práctica situaciones favorecedoras de aprendizajes transferibles. Con la finalidad de desarrollar la formación en evaluación, por su importancia transversal y sus posibilidades para favorecer el aprendizaje autónomo y la autorregulación, se han diseñado y analizado dos juegos de simulación para mejorar la alfabetización evaluadora: "Un día con Eva” y "EVONG: Evaluación en Acción”. Mediante un cuestionario y entrevistas grupales, se ha recogido información de 131 estudiantes de un Grado en Educación Primaria con la finalidad de conocer sus percepciones y valoraciones sobre estos recursos, especialmente sobre su utilidad educativa y sus posibilidades de transferencia. Tras el análisis de los datos cuantitativos y cualitativos, los resultados de la investigación reflejan que la principal competencia que se puede desarrollar es la toma de decisiones activa, favorecida por la comprobación y reflexión de las consecuencias que conlleva. El realismo y cercanía de las situaciones propuestas se ha percibido como un elemento facilitador para la generalización de los aprendizajes. A pesar de estos beneficios, la motivación real por superar las dinámicas, el esfuerzo docente para la creación de estos recursos -muy alejados de las producciones comerciales-, la necesidad de acciones formativas para profundizar o el cierto desencanto de los jugadores intensivos, siguen siendo aspectos de discusión.
\end{abstract}

Palabras clave: Evaluación; Educación superior; Juego de simulación; Videojuego; Enseñanza asistida por ordenador.

The increasing use in Higher Education of serious video games, that is, those with educational intentions, is justified by the experiential enrichment of the students. Within these, in this work we focus on simulation games, recreations of reality that allow us to experience transferable learning situations from practice. In order to develop the training in assessment, due to its transversal importance and its possibilities to promote autonomous learning and self-regulation, two simulation games have been designed and analysed to improve the assessment literacy: "A day with Eva" and "EVONG : Assessment in Action ". Through a questionnaire and group interviews, information has been collected from 131 students of a Grade in Primary Education with the purpuse of knowing their perceptions and valuations about these resources, especially about its educational utility and its transfer possibilities. After the analysis of the quantitative and qualitative data, the results of the research show the main competence that can be developed is the active decision-making, favoured by the verification and reflection of the consequences that it entails. The realism and proximity of the proposed situations has been perceived as a facilitating element for the generalization of learning. Despite these benefits, the real motivation to overcome the dynamics, the teaching effort for the creation of these resources -far removed from commercial productions-, the need for training actions to deepen or the certain disenchantment of intensive players, remain discussion aspects.

Keywords: Assessment; Higher education; Simulation games; Video games; Computer assisted instruction.

*Contacto: miguel.gomez@uca.es

issn: $1989-0397$

www.rinace.net/riee/

https://revistas.uam.es/riee
Recibido: $\quad 21$ de noviembre de 2019

$1^{\text {a }}$ Evaluación: 21 de enero de 2020

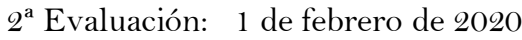

Aceptado: 7 de febrero de 2020 


\section{Introducción}

Hasta no hace demasiados años, hablar de "jugar a evaluar", sobre todo si nuestro propósito explícito es mejorar los conocimientos y habilidades relacionadas con la evaluación, habría sonado algo extraño, poco creíble e incluso ciertamente paradójico. No obstante, cada vez es más habitual en las instituciones educativas introducir metodologías relacionadas con convertir en juegos interacciones y contenidos didácticos que, en principio, no fueron pensados como tales. Es el conocido enfoque de gamificación o ludificación, que intenta explotar educativamente la motivación, el interés y la atracción de la mayoría de personas por los juegos (Teixes, 2015). Un enfoque que ha sido potenciado en las últimas décadas gracias a las posibilidades de las tecnologías para el desarrollo, la presentación e interacción con juegos electrónicos o videojuegos que han venido a aumentar y diversificar sus posibles aplicaciones a contextos formativos (Paradas-Castro, Raposo-Rivas y Martínez-Figueira, 2018).

Dentro de la amplia diversidad de videojuegos, tanto independientes como comerciales, en este trabajo nos centramos en una tipología concreta, los juegos serios o serious games, un aparente oxímoron que realmente esconde su intencionalidad específicamente educativa por encima de elementos lúdicos y que, especialmente en la Educación Superior, se está convirtiendo en una importante herramienta para mejorar la experiencia de los estudiantes (Nadolski et al., 2008) e incluso para rediseñar planes de estudio (Chen y Hoffman, 2017).

Son muchos los contenidos que se pueden trabajar, desde los distintos organismos que utilizan estos recursos, mediante los juegos serios (Gros-Salvat, 2009), pero a diferencia de la mayoría, en los que solo se presta especial atención a la evaluación en las actividades que se proponen durante su desarrollo (Serrano-Laguna et al., 2012), en nuestro caso, la evaluación se trata del propio contenido de los juegos diseñados. No solo porque la evaluación en la Educación Superior continúe siendo una de las grandes áreas donde es necesario proponer innovaciones y mejoras (Boud, 2006), sino porque mediante la formación en evaluación los estudiantes podrán comprender los principios básicos de la evaluación, las prácticas de retroalimentación, la naturaleza y significado de los criterios, podrán aplicar distintos enfoques y técnicas, y, lo más importante, podrán apreciar las relaciones de la evaluación con el aprendizaje (Price et al., 2012).

Asimismo, mediante la formación en evaluación se pueden desarrollar habilidades transversales de especial relevancia en los ámbitos académicos y profesionales, como la toma de decisiones, la creatividad, la resolución de problemas y estrategias para potenciar el aprendizaje autónomo de los estudiantes (Gómez-Ruiz, Rodríguez-Gómez e Ibarra-Sáiz, 2013). De ahí la especial relevancia e interés en fomentar en los estudiantes el aprendizaje en el ámbito de la evaluación, precisamente para permitirles que mediante la evaluación puedan aprender.

De esta forma, en este artículo se presenta una investigación que tiene como finalidades identificar las percepciones y describir las posibilidades de transferencia para 131 estudiantes de un Grado en Educación Primaria de dos juegos serios mediante los que se desarrollan conocimientos y competencias relacionadas específicamente con el ámbito de la evaluación. 


\section{Aprender a evaluar y evaluar para aprender}

El aprendizaje o la alfabetización en evaluación pretende ayudar a los estudiantes a comprender cómo funciona la evaluación en un contexto educativo (Ibarra-Sáiz y Rodríguez-Gómez, 2017). Este conocimiento sobre los procesos evaluativos en Educación Superior permite a los estudiantes estar más involucrados y rendir mejor en sus estudios (Brown, 2015), tener mayor confianza y competencia para realizar juicios razonados sobre sus propias producciones (Boud et al., 2010) y participar con garantías de forma razonada, consecuente y eficaz en cualquier fase de la evaluación universitaria (Smith et al., 2013). Además, cuando consideramos la evaluación como un aprendizaje, el alumnado puede desarrollar estrategias para regular su metacognición, monitorizando su propio aprendizaje y utilizando la retroalimentación para adaptar y ajustar lo que comprenden (Ibarra-Saíz y Rodríguez-Gómez, 2019).

De hecho, no es posible concebir la evaluación, en la actualidad y en el ámbito universitario, si no es como una realidad estrechamente ligada al aprendizaje, sin que importe excesivamente las preposiciones que acompañen esta relación (Hayward, 2015), pero siempre con el fin de extraer el máximo beneficio formativo para los implicados. Aprender de la evaluación, fundamentalmente desde la retroalimentación, que es considerada como el elemento más relevante en la enseñanza para desarrollar el aprendizaje autónomo y trabajar la autorregulación del alumnado (Hattie y Timperley, 2007). Y aprender con la evaluación, participando de manera activa en el diseño (criterios y planificación), la ejecución (mediante las modalidades participativas de la autoevaluación, evaluación entre iguales o evaluación colaborativa) o la decisión sobre la calificación (Quesada-Serra, García-Jiménez y Gómez-Ruiz, 2017).

La participación de los estudiantes es una parte crucial del aprendizaje en la evaluación, no solo por la aplicación práctica de los conocimientos en este ámbito, sino porque los protagonistas perciben que tienen un papel relevante en la construcción de su propio aprendizaje, pudiendo desarrollar un aprendizaje continuo y fomentando su responsabilidad (Penuel y Shepard, 2016). No obstante, y a pesar de los beneficios contrastados, en la actualidad es todavía frecuente que los estudiantes tengan un papel pasivo en el ámbito de la evaluación universitaria, un hecho que no solo limita el aprendizaje actual del alumnado, sino que compromete el aprendizaje a largo plazo (Boud y Falchikov, 2006).

Siendo, pues, conscientes de la relevancia formativa de la evaluación y, a su vez, de la importancia del desarrollo de la competencia evaluadora en los estudiantes, se ha buscado el diseño y creación de juegos serios, partiendo del enfoque de la gamificación en Educación Superior, mediante los que se puedan conocer los componentes fundamentales de los procesos evaluativos y con los que se pueda practicar en contextos realistas la toma de decisiones, el establecimiento de estrategias comparativas, la cumplimentación de instrumentos de evaluación y la reflexión de las consecuencias de las acciones propias en un entorno controlado. En coherencia con lo planteado, mediante los juegos se debería preparar a los estudiantes para la participación real en procesos de evaluación, a la vez que se fomentan habilidades valorativas que puedan extrapolar a sus estudios y a su vida. 


\section{El diseño de juegos de simulación}

Teniendo en consideración el enfoque y conociendo los distintos tipos de videojuegos existentes (Santos, Alloza y Escribano, 2018; Sedeño, 2010), se optó por el desarrollo de dos juego de simulación, es decir, juegos en los que se modela un sistema consistente con la realidad donde los jugadores pueden explorar, interactuar con los objetos y personas, comprobar su hipótesis e ideas, así como tomar decisiones relevantes en el marco de actividades formativas. Dentro de esta tipología, se suele distinguir entre simulaciones de alta fidelidad, donde se reproducen situaciones particulares en las que se espera que los estudiantes puedan participar, y simulaciones de baja fidelidad, donde se pretende trabajar las situaciones de forma más global y conceptual, eliminando variables extrañas y pudiendo experimentar distintas perspectivas comparándolas con diversas realidades (Squire, 2003).

De esta forma, los juegos serios que simulan la realidad se convierten en una útil herramienta para promover aprendizajes y transferir los conocimientos adquiridos, estimulando la participación en escenarios virtuales, generando expectativas y la voluntad de interacción (Poy-Castro, Mendaña-Cuervo y González, 2015). Así, se podría considerar evidente que jugando a simulaciones de la realidad, se involucra de manera profunda a los individuos en el contexto recreado, algo que facilita la comprensión y el aprendizaje de las estrategias puestas en práctica, a través de la modificación de modelos mentales que permiten un aprendizaje totalmente vivencial (Zamora-Enciso, 2010).

Realmente, si reflexionamos un poco, podemos pensar que la simulación y el aprendizaje están más unidos de lo que podría parecer en el proceso de enseñanza, aprendizaje y evaluación. De hecho, gran cantidad de las tareas de evaluación en la Educación Superior están basadas en entidades de simulación, como por ejemplo, los casos prácticos. Concretamente, en los juegos de simulación, los jugadores se enfrentan a retos que reproducen modelos simplificados de la realidad, tomando decisiones y experimentando en un entorno seguro, siendo a partir de la alternancia entre aciertos y errores cuando se logra mayores cotas de aprendizaje (Urquidi-Martín, 2014).

Si nos centramos en el nivel de diseño de los juegos, de manera general, cualquier proceso de gamificación se compone de tres elementos fundamentales: las mecánicas, las dinámicas y la estética (Teixes, 2015). En el caso específico de los juegos serios y las simulaciones en el ámbito educativo, se deben tener en consideración diferentes factores, como el contexto en el que el juego tiene lugar, los atributos particulares del grupo de estudiantes, el mundo de representación interna (modo de representación, interacción, nivel de inmersión y fidelidad) y las características pedagógicas del proceso de aprendizaje (De Freitas y Oliver, 2006).

De una manera más exhaustiva, y revisando las metodologías existentes para la creación de juegos serios, Buchinger y da Silva (2018) identifican una lista de características a considerar en diseño de este tipo de juegos entre las que se incluyen: Interacciones entre jugadores, sincronización, roles, recursos, puntuación, retos, recompensas o la inteligencia artificial a utilizar. Sin embargo, en el diseño de nuestros juegos de simulación para desarrollar la formación en evaluación, se tuvo como referencia la metodología EMERGO para el desarrollo de juegos serios basados en escenarios (Nadolski et al., 2008). Desde este enfoque, se debe comenzar una primera etapa de análisis donde se consideren aspectos del juego a desarrollar como el contexto, el 
contenido, el progreso de los estudiantes o el soporte. En la segunda etapa, comienza la fase de diseño en sí, donde se describen los escenarios, las actividades y los recursos necesarios. Posteriormente, se continuarían con las etapas de desarrollo, implementación y evaluación del juego.

\section{Juegos de simulación para aprender a evaluar: "Un día con Eva” y "EVONG: Evaluación en Acción”}

Con la finalidad de desarrollar la competencia evaluadora en los estudiantes universitarios, un equipo de docentes, acompañados por un diseñador gráfico y un programador informático, diseñaron y crearon dos juegos de simulación que permitieran interiorizar y practicar conceptos fundamentales del ámbito evaluativo. Los docentes universitarios, sin experiencia previa en la creación de juegos serios, trabajaron en ambos casos durante meses para establecer un guion totalmente explícito de lo que serían las futuras aventuras gráficas, creando la historia, la ambientación, la interrelación de escenas, personajes y objetos y, sobre todo, la coherencia de las actividades a realizar para la consecución de los objetivos formativos previstos, todo ello teniendo en consideración las posibilidades y limitaciones de la plataforma elegida para su edición.

De manera complementaria, el diseñador gráfico iba realizando pruebas hasta encontrar la estética más adecuada para las caracterizaciones de cada juego. Una vez que el estilo era aceptado, se comenzaba con la creación de los dibujos que harían realidad tanto los escenarios concretos, como los personajes intervinientes, así como los recursos y objetos con los que se interaccionaría. Finalmente, el informático pudo construir los juegos, ordenando y sistematizando las opciones, tareas y elementos intervinientes que originalmente se propusieron en el guion, con lógicas e inevitables modificaciones durante el proceso de desarrollo y compilación.

Desde un plano más técnico, los productos finales, que en las siguientes páginas se describen detalladamente, han sido dos aventuras gráficas del tipo point and click en 2D para escritorio, cuyo único requisito imprescindible para poder ejecutarse es tener instalado y actualizado el software gratuito multiplataforma "Java". Las simulaciones han sido desarrolladas con la plataforma "eAdventure", un conocido motor para la creación de videojuegos educativos que recientemente ha sido modificado y reiniciado con la denominación "uAdventure", tras combinar la experiencia de herramientas comerciales de juegos y herramientas educativas personalizadas (Pérez-Colado et al., 2017).

Concretamente, los juegos de simulación que se presentan detalladamente a continuación, se denominan "Un día con Eva" y "EVONG: Evaluación en Acción", creados en el contexto de sendos proyectos de investigación: del Proyecto DevalSimWeb ${ }^{1}$ el primero y del Proyecto DevalS ${ }^{2}$ el segundo. Estos juegos se utilizaron

\footnotetext{
${ }^{1}$ Proyecto DevalSimWeb: Desarrollo de competencias profesionales a través de la evaluación participativa y la simulación utilizando herramientas web $\left(\mathrm{N}^{\mathrm{o}}\right.$ DCIALA/19.09.01/11/21526/264-773/ALFAIII(2011)-10). Financiado por la Comisión Europea.

2 Proyecto DevalS: Desarrollo de la e-Evaluación sostenible. Mejora de la competencia evaluadora de los estudiantes universitarios mediante simulaciones virtuales. Referencia: EDU2012-31804. Financiado por el Ministerio de Economía y Competitividad.
} 
en estos proyectos como elementos clave para el diseño e implementación de programas formativos orientados a favorecer la adquisición de la competencia evaluadora en los participantes.

\subsection{Un día con Eva}

El juego "Un día con Eva" es una aventura gráfica en tercera persona que fue desarrollada durante 2014 y que se puede descargar de manera gratuita, tanto en versión española como inglesa, desde su página web: http://eva.evalfor.net/.

El argumento gira en torno a Eva (nombre elegido por su evidente evocación a su temática sobre evaluación), una estudiante universitaria que, como todas las personas, tiene que tomar numerosas decisiones a lo largo de su vida. El juego se sitúa en un día cualquiera para Eva en el que tendrá que ir tomando una serie de decisiones en los seis escenarios que componen el juego (habitación de Eva, Campus universitario, pasillo de la facultad, sala de estudio, comedor y aula). La estructura completa del juego se puede observar en la figura 1. Su duración estimada es de 25 a 30 minutos.

En "Un día con Eva" los jugadores deben realizar una serie de actividades, dirigiendo a su protagonista e interactuando con distintos objetos y personas, que supondrán un primer acercamiento al ámbito de la evaluación y mediante el cual se pretende conseguir en los estudiantes una reflexión argumentada sobre la relevancia de la evaluación en la vida cotidiana, así como explicitar algunos de los componentes fundamentales de la evaluación, como el establecimiento de criterios, la comparación, la valoración y la toma de decisiones, partiendo de aspectos tan cercanos como qué medio de locomoción utilizar para desplazarnos, cómo realizar mis trabajos universitarios, qué almorzar o si es conveniente salir esa noche con los amigos.

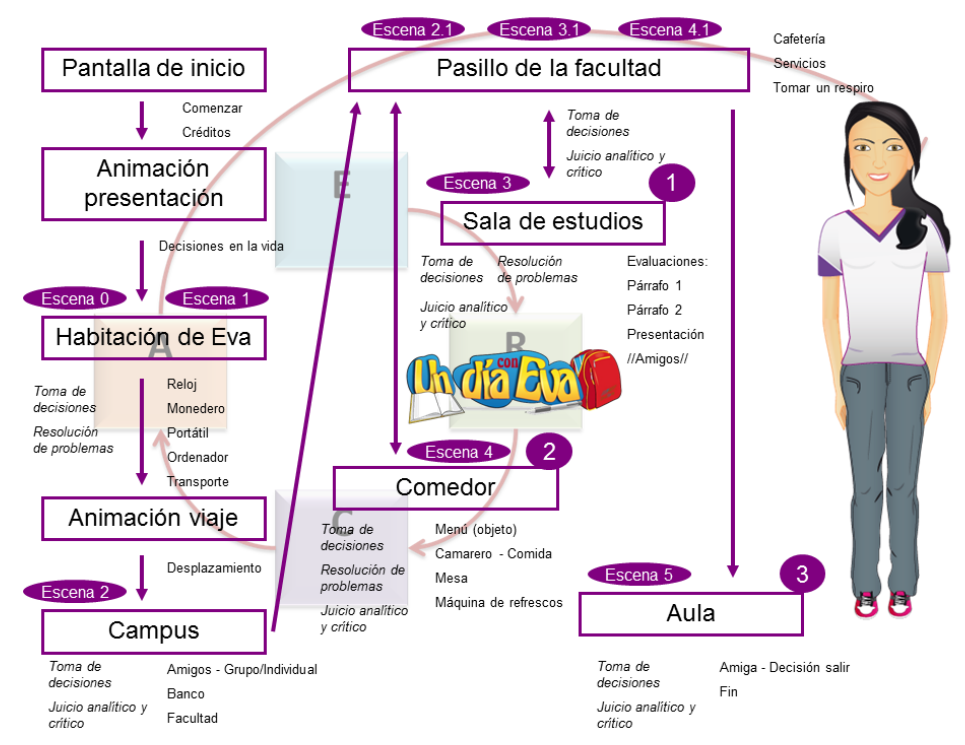

Figura 1. Estructura resumen del juego "Un día con Eva" Fuente: Elaboración propia.

De forma explícita, las competencias que se propusieron trabajar en los jugadores con el diseño y creación del juego "Un día con Eva” son las siguientes:

- Dar respuesta a situaciones cotidianas mediante el pensamiento crítico y el juicio analítico. 
- Tomar decisiones consecuentes con los criterios de evaluación.

- Resolver situaciones problemáticas mediante la valoración de distintas posibilidades.

- Establecer criterios y argumentos reflexionados y fundamentados.

- Desarrollar respuestas basadas en el razonamiento y la creatividad.

\subsection{EVONG: Evaluación en Acción}

El juego "EVONG: Evaluación en Acción” es una aventura gráfica en primera persona, es decir, que no existe un protagonista, sino que es el jugador el propio protagonista del juego, cuya última versión se publicó en el año 2016. Al igual como en el anterior caso, se puede descargar de manera gratuita, tanto en idioma español como inglés, desde su página web: http://evong.evalfor.net/.

El enfoque de este juego es distinto al de su antecesor, ya que se ambienta en un contexto laboral concreto, en nuestro caso, y de ahí su denominación, en una ONG (Organización No Gubernamental) ficticia que se denomina "InterAcción". Además, organizado desde tres perfiles diferenciados que se pueden seleccionar en cualquier orden: Coordinador de Economía y Calidad, Coordinador de Proyectos y Coordinador de Relaciones Institucionales. Realmente cada perfil es como un pequeño juego en sí mismo, con comienzo y final, en el que hay que realizar distintas tareas evaluativas diferenciadas según el tipo de coordinación, pero siempre dentro de una misma ambientación: la gestión de la ONG. El tiempo de juego de cada perfil es de aproximadamente 20 minutos y en todos los casos, la correcta consecución de las distintas actividades se refleja en la variable "Índice de éxito del área".

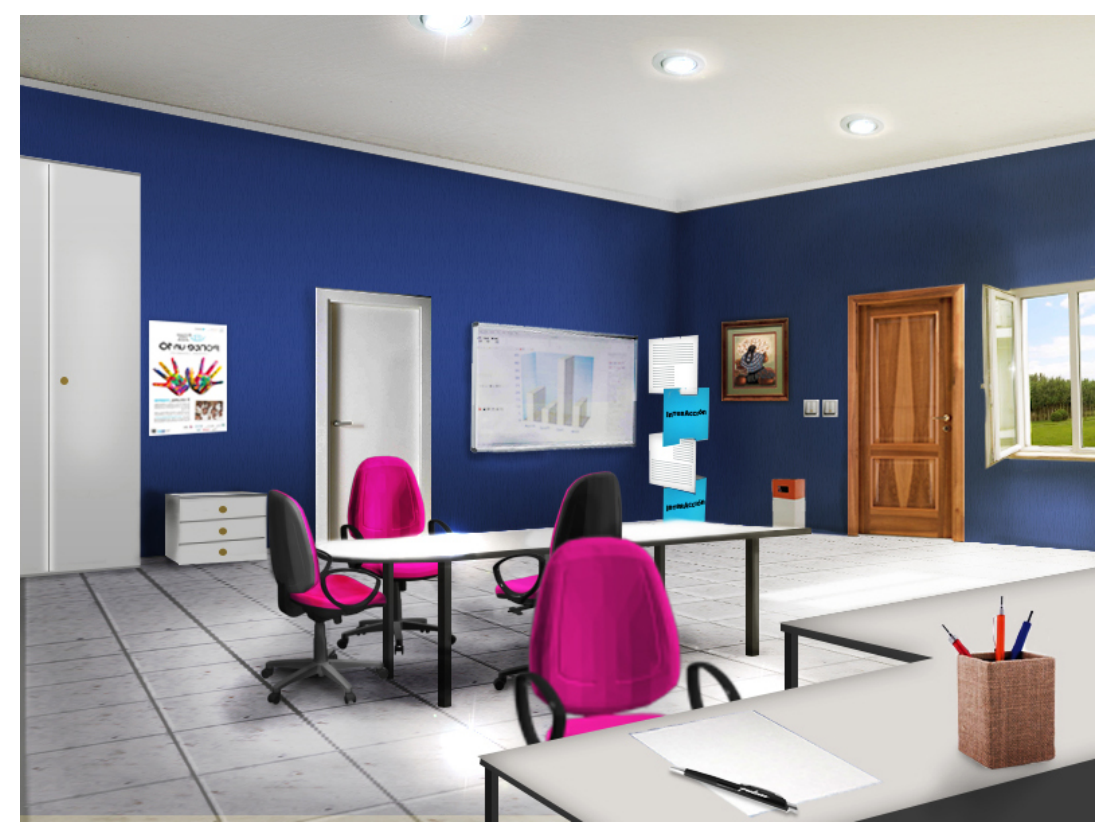

Figura 2. Escenario principal del juego "EVONG: Evaluación en Acción" Fuente: Elaboración propia. 
A diferencia de "Un día con Eva", "EVONG: Evaluación en Acción” es un juego de mayor duración, más formal, con lecturas más extensas y tareas más complejas y específicas con el que se pretende aplicar los conceptos trabajados anteriormente sobre evaluación, realizar valoraciones ajustadas a evidencias y tomar decisiones razonadas orientadas a la consecución de metas. En la figura 2, se puede observar el escenario central de la globalidad del juego, la sala principal de la oficina de la ONG "InterAcción".

Respecto a las competencias que se intentan trabajar con la interacción con este juego son las siguientes:

- Análisis de la información: Seleccionar, revisar y organizar la información disponible para identificar y extraer las ideas más relevantes y pertinentes.

- Aplicación de conocimientos relacionados con la evaluación: Utilizar y poner en práctica el conocimiento adquirido (conceptos, principios, procedimientos, actitudes) para el desarrollo de actividades y tareas académicas y profesionales.

- Aprendizaje autónomo: Conocer y autovalorar las propias necesidades formativas, determinar objetos de aprendizaje y planificar, gestionar y ejecutar estrategias educativas que faciliten su logro.

\section{Objetivos}

Se enumeran a continuación los objetivos que han guiado la investigación desarrollada:

- Identificar el valor y la utilidad para los estudiantes de los juegos de simulación diseñados ("Un día con Eva” y "EVONG: Evaluación en Acción”) para su formación en evaluación en el ámbito de la Educación Superior, haciendo hincapié en la identificación de los aspectos positivos y negativos de los mismos.

- Comparar las percepciones de los jugadores sobre el diseño de los dos juegos para establecer elementos diferenciadores e identificar nexos comunes.

- Describir las posibilidades de transferencia de los aprendizajes de los juegos para los participantes, tanto para la vida real, como para las tareas académicas.

\section{Método}

\subsection{Procedimiento}

Tras la aparición del segundo juego, durante el curso académico 2016/2017, se diseñó una investigación descriptiva y comparativa para recoger información, tanto de carácter cuantitativo como cualitativo, que permitiera profundizar en las percepciones de los usuarios de juegos de simulación para su formación en evaluación. Con este fin, y en el contexto de una práctica universitaria para el análisis de recursos innovadores, se les planteó estudiantes de la asignatura "Innovación e Investigación Educativas" del Grado en Educación Primaria de la Universidad de Cádiz que se descargaran y jugaran individualmente a los dos juegos de simulación descritos: En primer lugar "Un día con Eva" y posteriormente "EVONG: Evaluación en Acción”.

Tras el periodo de interacción y experimentación con ambos juegos, los estudiantes debían cumplimentar de manera individual un cuestionario en el que se recogerían sus 
percepciones sobre la utilidad formativa de los juegos de simulación creados, utilizando las mismas preguntas e ítems a valorar tanto para "Un día con Eva" como para "EVONG: Evaluación en Acción", que permitieran en pasos posteriores analizar comparativamente estos dos recursos educativos.

Para finalizar y complementar la recogida de información, los estudiantes se agruparon presencialmente en equipos de trabajo para intercambiar sus opiniones y experiencias con los juegos de simulación mediante la realización de distintas entrevistas grupales. Estas entrevistas, que tenían como finalidad profundizar y contrastar las visiones individuales plasmadas en los cuestionarios, fueron desarrolladas autónomamente por cada equipo de trabajo siguiendo un mismo guion de preguntas y grabándose en formato audio. La duración total de la recogida de información fue de tres semanas.

\subsection{Participantes}

En esta investigación han participado como informantes un total de 131 estudiantes que estaban matriculados en el segundo curso del Grado en Educación Primaria de la Universidad de Cádiz. Del total de los estudiantes, 65 pertenecían al grupo A (49,6\%) y 66 al grupo C $(50,4 \%)$ del Grado. Era de especial interés para este estudio que el alumnado que jugara y analizara los juegos estuviera en los primeros años de su carrera universitaria y que esta fuera del ámbito de la educación para intentar relacionar explícitamente estos juegos de simulación con su utilidad formativa y su capacidad motivadora como recursos didácticos innovadores por parte de los propios receptores.

La edad media de los participantes se sitúa en los 20,41 años. La gran mayoría de los estudiantes tenían en el momento de la recogida de información entre 19 y 20 años, concretamente, un 53,4\% (70 personas) tenían 19 años y un 19,1\% (25) contaban con 20 años. Únicamente 7 estudiantes $(5,6 \%)$ habían cumplido o superado los 24 años, encontrando los valores máximos en 1 participante $(0,8 \%)$ con 42 años y otro con 51 años. Respecto a la segmentación de los informantes por sexo, encontramos una participación predominante de mujeres que alcanza el $71,8 \%$ (94), mientras que el restante $28,2 \%$ fueron hombres (37). En último lugar, se debe apuntar que los 131 estudiantes participantes se agruparon en 27 equipos de trabajo de entre 4 a 6 componentes para desarrollar las entrevistas grupales.

\subsection{Técnicas e instrumentos}

\subsubsection{Cuestionario "Análisis de los juegos de simulación 'Un día con Eva' y 'EVONG”"}

El cuestionario virtual realizado, anónimo y disponible en http://goo.gl/forms/GR6gn7ctZh, es una adaptación simplificada de otro utilizado en investigaciones anteriores con fines similares (Rodríguez-Gómez e Ibarra-Sáiz, 2014). En él, los estudiantes individualmente plasmaban sus opiniones y percepciones sobre cada uno de los juegos con los que interactuaron.

El cuestionario se divide en 3 bloques diferenciados:

- Bloque I: Perfil de los participantes, donde se pedía información sobre la edad, el sexo y el nombre del equipo de trabajo al que pertenecían.

- Bloque II: Cuestiones sobre el juego "Un día con Eva"

- Bloque III: Cuestiones sobre el juego "EVONG: Evaluación en Acción” 
Tanto el Bloque II como el Bloque III se componían de las mismas 7 preguntas, pero centrándose cada bloque en cada uno de los dos juegos analizados. En las tres primeras preguntas, los estudiantes debían mostrar su grado de acuerdo con afirmaciones de carácter general (interés, realismo, motivación y relevancia del juego), sobre la estética y jugabilidad (atractivo gráfico, facilidad de uso y suficiencia de la información aportada en el juego) y sobre los contenidos trabajados (conocimientos sobre evaluación, toma de decisiones, aprendizaje autónomo y pensamiento creativo). En todos los casos, la escala Likert utilizada para valorar las afirmaciones era: Nada de acuerdo, poco de acuerdo, algo de acuerdo, bastante de acuerdo, muy de acuerdo y totalmente de acuerdo.

En la cuarta pregunta se les consultaba a los jugadores de forma explícita si consideraban que los aprendizajes trabajados mediante los juegos de simulación que habían probado les podrían ser de utilidad para sus estudios o para su vida real. Las dos siguientes cuestiones eran preguntas abiertas donde se les pedía a los participantes que redactaran los aspectos positivos y negativos del juego que en cada uno de los bloques se estaba analizando. El cuestionario se cerraba con una última pregunta aglutinadora en la que se debía realizar una valoración global de cada juego en una escala de 1 (mínimo) a 10 (máximo).

El tiempo estimado para la cumplimentación del cuestionario virtual completo era de unos 15 a 20 minutos. Un fragmento de la apariencia del mencionado cuestionario “Análisis de los juegos de simulación 'Un día con Eva' y 'EVONG”' se puede apreciar en la Figura 3:

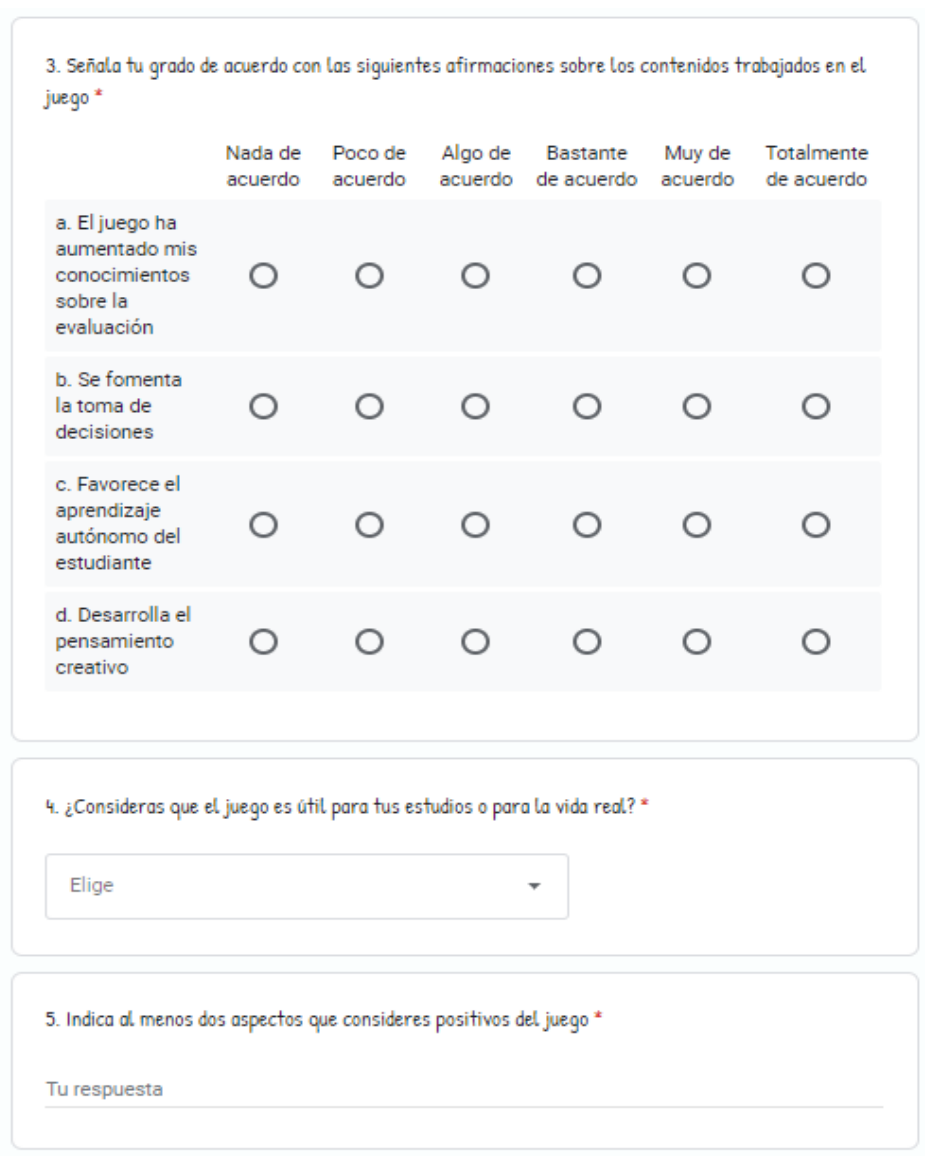

Figura 3. Captura de pantalla de un fragmento del cuestionario virtual utilizado Fuente: Recuperado de http://goo.gl/forms/GR6gn7ctZh 
6.3.2. Entrevista grupal: Análisis de innovaciones educativas sobre los juegos "Un día con Eva" y "EVONG"

Para completar la recogida de información se desarrollaron un total de 27 entrevistas grupales con sendos equipos de trabajo en los que se dividían, de 4 a 6 componentes, la totalidad de los estudiantes participantes en la investigación. Cada equipo realizó su entrevista de manera independiente, siendo igualmente grabadas cada uno de ellas en audio y posteriormente transcritas en texto para facilitar su análisis.

La duración de cada entrevista grupal ha variado sustancialmente, desde no alcanzar los 5 minutos, hasta llegar a la media hora de diálogo. La duración media la podemos situar en torno a los 8 ó 9 minutos. El guion que fue utilizado se componía de un total de 7 cuestiones mediante las que se intentaba profundizar sobre las siguientes temáticas:

1. Impresiones generales de los juegos y consideración como herramientas educativas útiles y motivadoras.

2. Posibilidades formativas de los juegos.

3. Consideración de los juegos como una innovación.

4. Transferencia de las competencias trabajadas a la vida real

5. Verdadera motivación en tener éxito en el juego o en aprender

6. Aspectos de mejora

7. Otros comentarios a destacar

Para concluir, ofrecemos en el cuadro 1 un resumen de las técnicas e instrumentos utilizados y descritos en este apartado:

Cuadro 1. Técnicas e instrumentos utilizados en la recogida de información

\begin{tabular}{lcc}
\hline \multicolumn{1}{c}{ TÉCNICA/INSTRUMENTO } & MODALIDAD & NúMERO \\
\hline $\begin{array}{l}\text { Cuestionario: Análisis de los juegos de simulación “Un día con } \\
\text { Eva" y "EVONG" }\end{array}$ & Individual & 131 \\
\hline $\begin{array}{l}\text { Entrevista grupal: Análisis de innovaciones educativas sobre } \\
\text { los juegos "Un día con Eva" y "EVONG" }\end{array}$ & $\begin{array}{c}\text { Grupal (4-6 } \\
\text { componentes) }\end{array}$ & 27 \\
\hline
\end{tabular}

Fuente: Elaboración propia.

\subsection{Análisis de datos}

Una vez concluido el procedimiento de recogida de información, se ha procedido al análisis de los datos textuales provenientes de las preguntas abiertas del cuestionario y de las entrevistas grupales, así como de los datos numéricos procedentes de los ítems cerrados del cuestionario sobre el grado de acuerdo con las afirmaciones propuestas y las valoraciones globales sobre los juegos. Dependiendo de su propia naturaleza cualitativa o cuantitativa se han seguido procedimientos de análisis diferenciados.

Por una parte, los datos cuantitativos (números o tratados como tales) del cuestionario se han analizado primeramente mediante el cálculo de estadísticos descriptivos como medias, desviaciones típicas y frecuencias. Posteriormente se procedió a realizar un análisis comparativo entre las respuestas obtenidas para cada uno de los juegos que culminaron con la realización de la prueba $\mathrm{T}$ de Student para muestras relacionadas para comprobar si las diferencias detectadas entre las valoraciones de ambos juegos eran 
estadísticamente significativas. Este análisis se ha realizado con la ayuda del paquete estadístico IBM SPSS Statistics en su versión 24.

Por otra parte, los datos cualitativos (textuales) se han analizado siguiendo el proceso general de análisis de datos cualitativos, un esquema general propuesto por Miles y Huberman (1994), según el cual en el análisis concurren tareas de reducción, disposición y transformación de los datos, así como la obtención y verificación de conclusiones a partir de la categorización de las unidades de información por tipo de significados (Rodríguez-Gómez y Gómez-Ruiz, 2010). Las categorías y subcategorías de análisis, detalladas en el siguiente apartado, se han establecido de forma emergente al examinar el contenido de la información, contrastándose mediante triangulación de investigadores.

\section{Resultados}

\subsection{Resultados del cuestionario}

\subsubsection{Valoración de la estética, jugabilidad, contenidos y aspectos generales de los juegos}

Como se comentó anteriormente, en las tres primeras preguntas del cuestionario sobre los juegos, los participantes debían establecer su grado de acuerdo (desde nada de acuerdo -1- hasta totalmente de acuerdo -6-) con un total de 11 afirmaciones sobre sus impresiones generales (1. El juego me ha parecido interesante; 2. La historia y situaciones son realistas; 3. Las actividades son motivantes y 4. El juego lo considero relevante), sobre la estética y jugabilidad (5. El juego me ha parecido atractivo gráficamente; 6. Es un juego sencillo e intuitivo de jugar y 7 . En la realización de actividades se ofrece información sobre los logros) y sobre los contenidos y competencias que se pretenden trabajar (8. El juego ha aumentado mis conocimientos sobre la evaluación; 9. Se fomenta la toma de decisiones; 10. Favorece el aprendizaje autónomo del estudiante y 11. Desarrolla el pensamiento creativo). En la figura 4 se ofrece un gráfico con las valoraciones medias de los estudiantes participantes con las mencionadas afirmaciones, tanto para el juego "Un día con Eva" como para "EVONG: Evaluación en Acción".

Realizando una primera lectura global de los resultados obtenidos, se puede comprobar que las afirmaciones con las que están más de acuerdo los estudiantes son la número 9 "Se fomenta la toma de decisiones" ( $\bar{x}=4,46$ y $\sigma=1,05)$ y la número 2 "La historia y situaciones son realistas" ( $\bar{x}=4,42$ y $\sigma=1,09)$. Estas dos sentencias, junto con la número 7 "En la realización de actividades se ofrece información sobre los logros" $(\bar{x}=$ 4,13 y $\sigma=1,16$ ), son las únicas que sobrepasan de forma global una valoración media de 4. Respecto a las afirmaciones con las que los jugadores están en menor medida de acuerdo, encontramos muy emparejadas la número 3 "Las actividades son motivantes" $\bar{x}$ $=3,28$ y $\sigma=1,15)$ y la número 8 "El juego ha aumentado mis conocimientos sobre evaluación" ( $\bar{x}=3,30$ y $\sigma=1,12)$.

Si realizamos un análisis comparativo de los resultados de cada uno de los juegos por separado, comprobamos que las afirmaciones más valoradas se corresponden con los resultados globales. En "Un día con Eva" las medias más altas las obtuvieron la número $2(\bar{x}=4,79$ y $\sigma=1,05)$ y la número $9(\bar{x}=4,76$ y $\sigma=1,02)$ y a la inversa en el caso de "EVONG: Evaluación en Acción”, la número 9 en primer lugar $(\bar{x}=4,15$ y $\sigma=1,08)$ y 
en segundo la número $2(\bar{x}=4,05$ y $\sigma=1,14)$. Respecto a las medias más bajas, en "Un día con Eva” encontramos la número $8(\bar{x}=3,43$ y $\sigma=1,07)$ y la número $3(\bar{x}=3,47$ y $\sigma$ $=1,09)$, mientras que en "EVONG: Evaluación en Acción" son la número $3(\bar{x}=3,09$ y $\sigma$ $=1,21)$ y la número 6 "Es un juego sencillo e intuitivo de jugar" ( $\bar{x}=3,14$ y $\sigma=1,37)$.

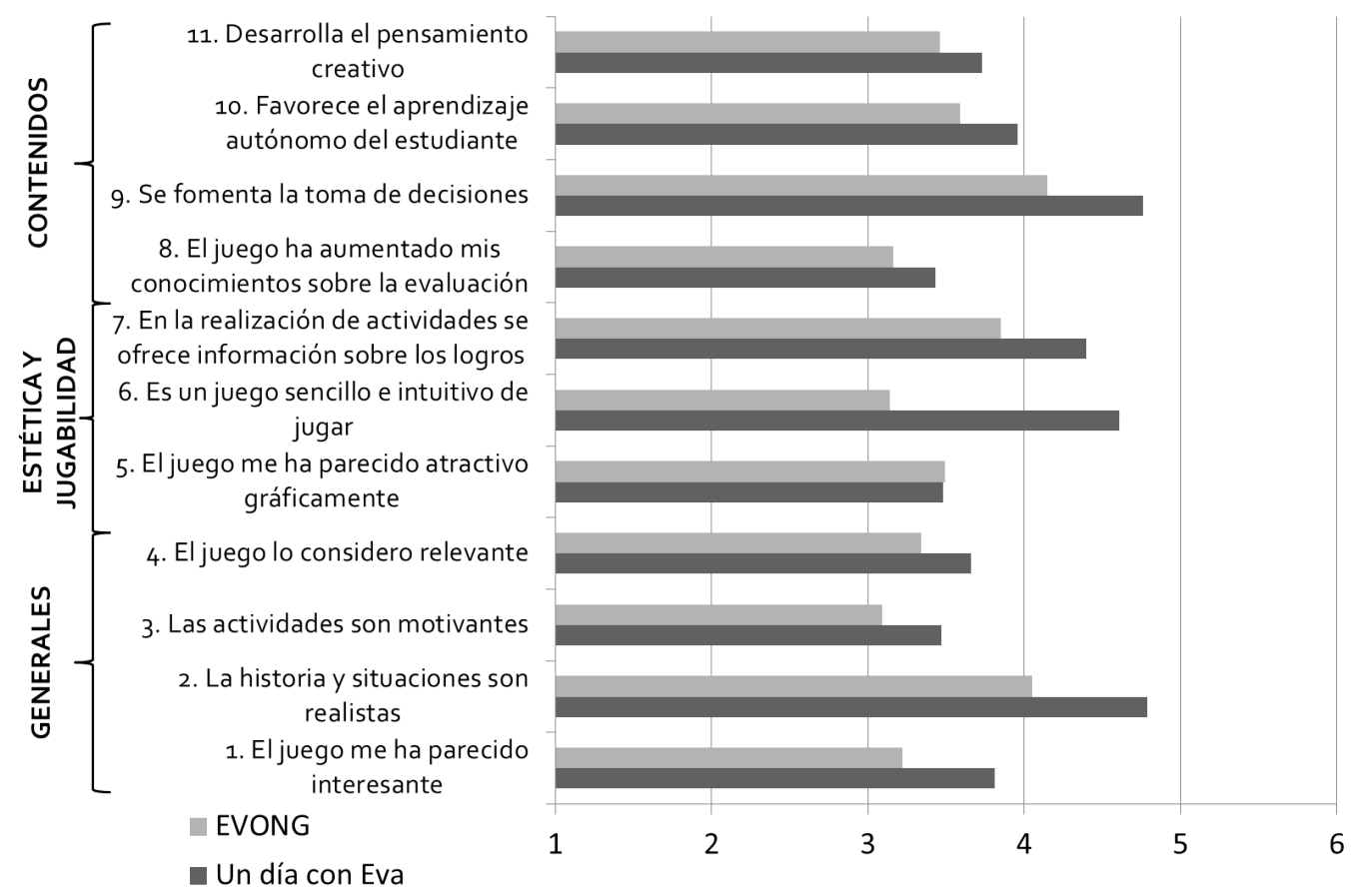

Figura 4. Valoraciones medias del grado de acuerdo con las afirmaciones propuestas en el cuestionario

Fuente: Elaboración propia.

Si se vuelve la mirada nuevamente a la figura 4, podremos apreciar que en todos los casos se valora claramente de manera más elevada al juego "Un día con Eva" menos en uno, la frase número 5 "El juego me ha parecido atractivo gráficamente" en el que "EVONG: Evaluación en Acción" es valorado ligeramente más alto de forma media $(+0,01)$. La afirmación en la que existe una mayor diferencia $(+1,47)$ entre el grado de acuerdo de un juego a otro, es la número 6 que para "Un día con Eva" es el tercer mejor valor $(\bar{x}=4,61$ y $\sigma=1,10)$, mientras que como se acaba de describir más arriba es el segundo peor valor en "EVONG: Evaluación en Acción”.

Las evidentes diferencias entre los juegos se reflejan igualmente en la valoración media de todas las afirmaciones, que en el caso de "Un día con eva" es $\bar{x}=4,01$ y en el caso de "EVONG: Evaluación en Acción” de $\bar{x}=3,50$. No obstante, se ha realizado la prueba T de Student para muestras relacionadas con las medias del grado de acuerdo a cada frase para comprobar si estas diferencias son estadísticamente significativas. Como se puede apreciar en el cuadro 2 , el cálculo realizado arroja que $\mathrm{p}=0,001$ menor que $\alpha=0,01$, por lo que se puede corroborar la existencia de diferencias significativas entre las valoraciones realizadas a los juegos analizados. 
Cuadro 2. Resultados de la T de Student de las afirmaciones de los juegos

\begin{tabular}{|c|c|c|c|c|c|c|c|c|}
\hline & \multirow[t]{2}{*}{ Media } & \multirow[t]{2}{*}{ DESV.TíP. } & \multirow{2}{*}{$\begin{array}{l}\text { ERROR } \\
\text { TÍP. } \\
\text { MEDIA }\end{array}$} & \multicolumn{2}{|c|}{$\begin{array}{l}\text { 99\% INTERVALO DE } \\
\text { CONFIANZA PARA LA } \\
\text { DIFERENCIA } \\
\end{array}$} & \multirow[t]{2}{*}{$\mathrm{T}$} & \multirow[t]{2}{*}{ GL } & \multirow[t]{2}{*}{$\begin{array}{c}\text { SIG. } \\
\text { (BILATERAL) }\end{array}$} \\
\hline & & & & INFERIOR & SUPERIOR & & & \\
\hline $\begin{array}{l}\text { Eva - } \\
\text { EVONG }\end{array}$ &, 50545 & ,38085 & , 11483 &, 14152 & ,86938 & 4,402 & 10 & ,001 \\
\hline
\end{tabular}

Fuente: Elaboración propia.

\subsubsection{Percepción sobre la utilidad de los juegos}

En la cuarta pregunta del cuestionario, los jugadores debían reflejar su percepción sobre si consideraban de utilidad los juegos para aplicar los conceptos y habilidades trabajados a sus estudios, a su vida real, a ambos o a ninguno. Nuevamente podemos comprobar las diferencias existentes entre ambos recursos, ya que un $74 \%$ (97 personas) consideraron de utilidad "Un día con Eva" tanto para los estudios como para la vida real, mientras que en el caso de "EVONG: Evaluación en Acción" el porcentaje no llega a la mitad de los participantes, con un 48,1\% (63). En el extremo contrario, hallamos que los jugadores que no consideran útil a "EVONG: Evaluación en Acción" asciende al 14,5\% (19), siendo únicamente un 6,9\% (9) los que no le encuentran utilidad a "Un día con Eva". En la figura 5 se muestran las frecuencias sobre la utilidad percibida por los 131 jugadores participantes en la investigación.

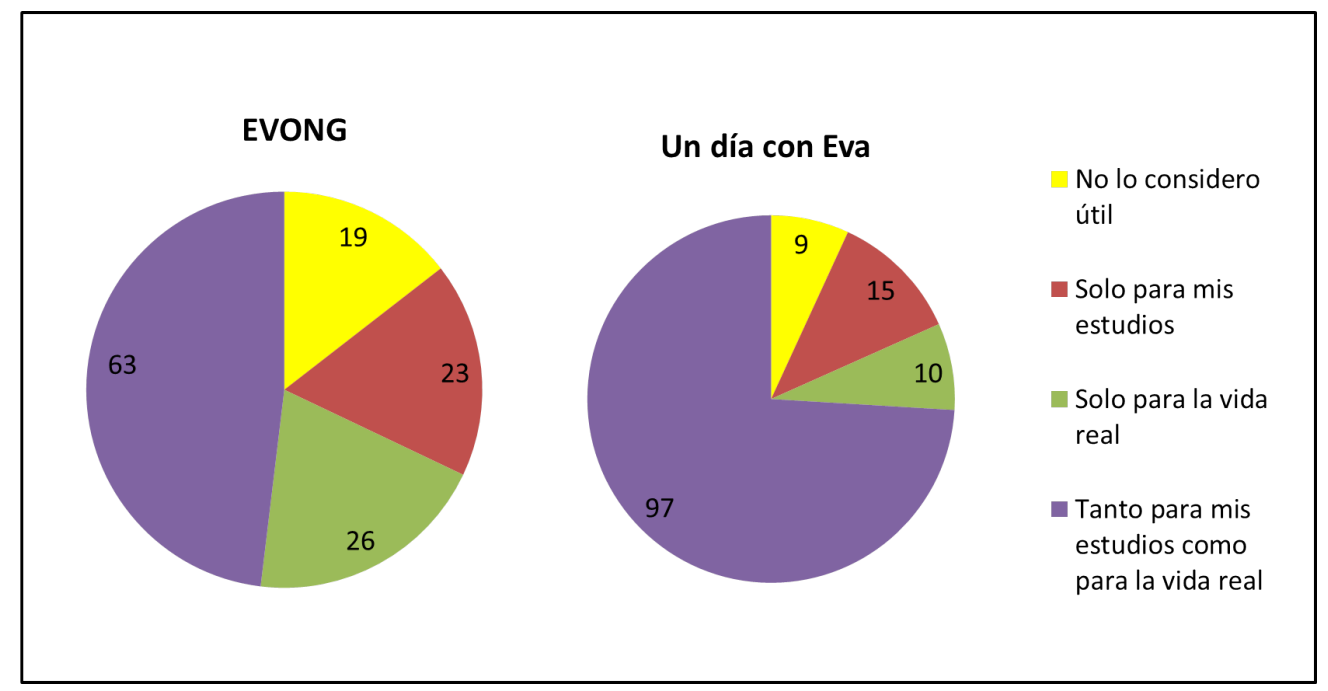

Figura 5. Frecuencia de la utilidad percibida por los estudiantes de los juegos analizados Fuente: Elaboración propia.

\subsubsection{Aspectos positivos y negativos de los juegos}

De manera abierta, se les pidió igualmente a los estudiantes que escribieran los principales aspectos tanto positivos como negativos que habían detectado después de jugar a "Un día con Eva” y a "EVONG: Evaluación en Acción”. En primer lugar, sobre los aspectos positivos percibidos, se ofrece en el cuadro 3 la categorización construida a partir de las respuestas y la frecuencia de cada categoría para los dos juegos objeto de análisis. 
Cuadro 3. Aspectos positivos de los juegos para los participantes

\begin{tabular}{|c|c|c|c|}
\hline Categoría & $\begin{array}{l}\text { UN DíA CON EVA } \\
(\mathrm{F})\end{array}$ & $\begin{array}{c}\text { EVONG: } \\
\text { EVALUACIÓN EN } \\
\text { ACCIÓN (F) }\end{array}$ & TOTAL \\
\hline $\begin{array}{l}\text { Fomenta la toma de decisiones de manera } \\
\text { reflexiva y las consecuencias que conllevan }\end{array}$ & 87 & 59 & 146 \\
\hline Es un juego cercano que refleja la realidad & 43 & 33 & 76 \\
\hline Juego entretenido y con un diseño atractivo & 30 & 33 & 63 \\
\hline $\begin{array}{l}\text { Mejora la organización, responsabilidad y } \\
\text { autonomía de los estudiantes }\end{array}$ & 17 & 26 & 42 \\
\hline Sirve para formarse y comprender la evaluación & 22 & 11 & 33 \\
\hline Genera motivación & 8 & 2 & 10 \\
\hline Fomenta el trabajo cooperativo & 6 & 3 & 9 \\
\hline Permite conocer el trabajo de las ONGs & $\mathrm{O}$ & 4 & 4 \\
\hline Mejora la puntualidad & 3 & $\mathrm{O}$ & 3 \\
\hline Otros & 2 & 4 & 6 \\
\hline
\end{tabular}

Fuente: Elaboración propia.

Coherentemente con las afirmaciones anteriormente analizadas, el aspecto positivo más destacado en ambos juegos, es que se trabaja la toma de decisiones de manera reflexiva y las consecuencias que conllevan. Hasta el $66,4 \%$ (87 personas) así lo han percibido en el juego "Un día con Eva” y el 45\% (59) en "EVONG: Evaluación en Acción”. A continuación se ofrece un ejemplo, de cada uno de los juegos, de respuestas de los estudiantes incluidas en esta categoría de análisis:

Hace que pensemos en la repercusión que tendrán nuestras decisiones y una vez que las hemos tomado, nos hace pensar si verdaderamente hemos hecho lo que pensábamos en un principio. $\left(\mathrm{P}_{26}\right)$

Valora la importancia de la toma de decisiones laborales y las consecuencias que estas tienen en la empresa. (PO5)

También alineado con las afirmaciones, surge, en segundo lugar que son juegos cercanos capaces de reflejar fielmente la realidad. 43 personas lo mencionan para "Un día con Eva" (32,8\%) y 33 para "EVONG: Evaluación en Acción" (25,2\%). Algunos fragmentos significativos de esta categoría pueden ser los siguientes:

Es muy real y te puedes identificar con el juego en todos los aspectos. (P91)

Otro aspecto positivo que puedo resaltar es la naturalidad de las situaciones, son muy reales y nos puede pasar a todos en un futuro. (Po7)

Ya en menor medida, pero con suficiente relevancia, los jugadores consideran positivos tanto el diseño atractivo (24\% del total) de los juegos como que sirven para formarse y comprender mejor la evaluación (12,6\%), dos aspectos con los que menos acuerdo mostraron en la valoración de las afirmaciones. También que mejora la organización, responsabilidad y autonomía de los estudiantes ha sido otro aspecto positivo mencionado en diversas ocasiones (16\%). Una muestra de estas tres categorías se ofrece a continuación:

Como aspectos positivos destaco lo atractivo que es gráficamente y lo real que parecen los textos que hay en el juego. (P75)

Sirve de mucha ayuda para comprender mejor la evaluación. (P108)

Fomenta la autonomía, responsabilidad y madurez en el alumno. (Po2)

Respecto a los aspectos negativos descritos en el cuestionario, como se puede apreciar en el cuadro 4, existe una mayor especificidad dependiendo del juego. En "Un día con Eva" 
el $39,7 \%$ de los jugadores (52) opinan que lo peor es su jugabilidad y la calidad de sus diseños, el 22,1\% (29) consideran por su parte que es excesivamente sencillo y lento, y el mismo porcentaje que este juego dispone de unas opciones limitadas para la toma de decisiones.

Cuadro 4. Aspectos negativos de los juegos para los participantes

\begin{tabular}{lccc}
\hline \multicolumn{1}{c}{ Categoría } & $\begin{array}{c}\text { UN Día CON } \\
\text { Eva (F) }\end{array}$ & $\begin{array}{c}\text { EVONG: EvaluACión } \\
\text { EN ACCión (F) }\end{array}$ & TotaL \\
\hline $\begin{array}{l}\text { La jugabilidad y la calidad de los diseños son } \\
\text { mejorables }\end{array}$ & 52 & 44 & 96 \\
Resulta monótono y poco motivador & 27 & 56 & 83 \\
$\begin{array}{l}\text { Ausencia de orientaciones y aclaraciones en el } \\
\quad \text { juego }\end{array}$ & 7 & 41 & 48 \\
Excesivamente sencillo, lento e intuitivo & 29 & 12 & 41 \\
Opciones limitadas en la toma de decisiones & 29 & 6 & 35 \\
Corta duración del juego & 25 & 0 & 25 \\
Poco realismo de algunos movimientos o escenas & 16 & 7 & 23 \\
Exceso de lecturas en el juego & 9 & 12 & 21 \\
No se ajusta a la edad de los destinatarios & 5 & 0 & 5 \\
No se refuerza suficientemente las ejecuciones & 3 & 0 & 3 \\
$\quad$ correctas & 2 & 5 & 7 \\
\hline Otros
\end{tabular}

Fuente: Elaboración propia

En "EVONG: Evaluación en Acción”, 56 personas (42,7\%) apuntan a que resulta monótono y poco motivador, 44 jugadores (33,6\%) señalan también la jubabilidad y la calidad de sus diseños y 41 (31,3\%) que se trata de un juego sin suficientes orientaciones y aclaraciones que faciliten su interacción.

Con la intención de ilustrar la construcción de estas categorías, se ofrece a continuación un ejemplo de los cinco aspectos negativos más referenciados en términos generales (ordenados descendentemente) y que han sido comentados en el anterior párrafo:

Por un lado, no te detalla bien lo que tienes que hacer, tienes que descubrirlo por tu cuenta. Deberían de haber más salas y más objetos que recoger y sobre todo más comunicación con otras personas de la misma empresa. (P31)

Que en muchas ocasiones se hace monótono porque hay que seguir unas pautas tal y como viene. (P66)

Uno de los aspectos negativos es que se trata de un juego bastante difícil de comprender, en el que no se especifica bien qué es lo que tenemos o no que hacer. (P64)

Puede resultar bastante sencillo de resolver. ( $\mathrm{P} 12)$

Me ayuda a tomar decisiones, como he dicho antes, pero a su vez me limita a tomarlas ya que tengo que escoger lo que el juego crea más conveniente $(\mathrm{P} 42)$

\subsubsection{Valoración global de los juegos}

Para concluir la exposición de resultados sobre el cuestionario de análisis de los juegos, nos centramos en la última pregunta de la que se componía este instrumento, una valoración global de cada uno de los juegos en una escala de 1 (mínimo) a 10 (máximo). Continuando con la tendencia en las contestaciones anteriores, "Un día con Eva” obtiene una valoración media superior en 1,19 puntos $(\bar{x}=7,18$ y $\sigma=1,19)$ a la conseguida por “EVONG: Evaluación en Acción” ( $\bar{x}=5,99$ y $\sigma=1,71$ ).

De hecho, si realizamos un cálculo comparativo entre las valoraciones de los jugadores a ambos juegos, hallamos que el 63,4\% de los participantes (83) ha valorado con mejor 
puntuación a "Un día con Eva", mientras que solo el 14,5\% (19) ha hecho lo propio con "EVONG: Evaluación en Acción”. 29 personas (22,1\%) los han valorado exactamente igual. En la Figura 6 se pueden comprobar la frecuencia de las valoraciones globales otorgadas a cada uno de los juegos, evidenciándose esta vez, de una manera gráfica, la existencia de una mayoría de valoraciones positivas a "Un día con Eva".

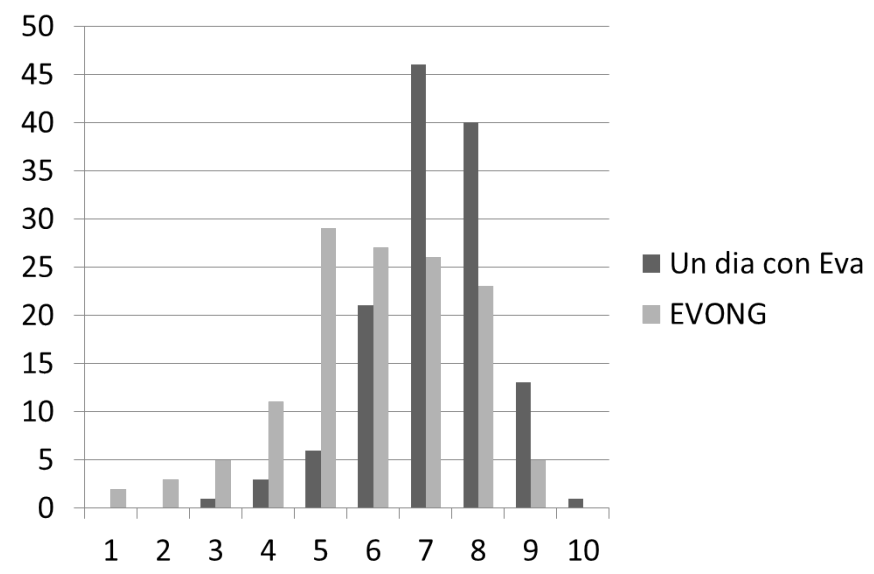

Figura 6. Frecuencia de las valoraciones globales a los juegos analizados Fuente: Elaboración propia.

\subsection{Resultados de la entrevista grupal}

\subsubsection{Utilidad, motivación e innovación de los juegos}

En la primera pregunta del guion de las entrevistas grupales, se les interrogaba a los participantes si creían que los juegos eran herramientas educativas útiles. De las 27 entrevistas, en 22 de ellas $(81,5 \%)$ se concluyó que sí tenían utilidad, mayormente porque se fomenta la toma de decisiones y la reflexión (9 referencias) y porque permite enfrentarse a situaciones reales (5). En el resto de las entrevistas, se consideraba que la utilidad concreta dependería de la edad de los receptores o de la mejora de ciertos aspectos de los juegos. A continuación ofrecemos dos fragmentos sobre las contestaciones de la utilidad de las principales categorías comentadas:

\footnotetext{
Nos parece una herramienta útil, pues el alumno comprueba mediante un juego que la toma de decisiones es importante a lo largo del día y que hasta las decisiones más pequeñas pueden producir grandes cambios. (G13)

Si se les da una situación real los niños pasan, pero con un juego al que esté exponiéndose la misma situación resulta más entretenido. (G05)
}

Sobre si es una herramienta motivadora, 16 referencias $(59,3 \%)$ apuntan a que sí lo es, por ser un recurso entretenido y divertido (7) así como novedoso y actual (6). No obstante, en 4 entrevistas se ponían en serias dudas su capacidad motivadora y en otras 2 se habló explícitamente de que no les motivó excesivamente por jugarse individualmente y porque no entretienen tanto como los juegos comerciales. Ejemplos de la categoría positiva se reflejan en las siguientes líneas:

Sirven para motivar a los alumnos a querer aprender ya que es una dinámica distinta y resultan divertidos. (G01)

Además nos parece una actividad motivadora pues se cambia la metodología tradicional (libros, actividades, pizarra...) y eso siempre hace que los alumnos tengan más ganas de aprender. (G24) 
No obstante, cuando se intenta profundizar sobre si esa motivación que provocan los juegos se debe principalmente a tener éxito en el mismo sin que importe la adquisición de aprendizajes, los grupos de estudiantes muestran mayor diversidad de percepciones. En 7 entrevistas $(25,9 \%)$ se concluye que sí, que la motivación está en superar el propio juego dentro de las reglas que se proponen. En 11 de los equipos (40,7\%) se plantean que esto dependerá de si existe reflexión sobre lo que se ha realizado o si hay orientaciones adecuadas de un docente. Por último, en 8 grupos (29,6\%) se referencia que esta motivación por tener éxito se traducirá automáticamente en aprendizaje y que este aprendizaje está muy por encima de su carácter lúdico. Dos ejemplos de las contestaciones comentadas se pueden leer a continuación:

To sí pienso que juegan con el propósito de tener éxito en el juego, porque cuando empiezas el juego de "Un día con Eva", a la hora de tomar decisiones seleccionamos las opciones más correctas cuando en la vida real lo más probable es que no la elijamos... para terminar el juego de la manera más coherente y adecuada. (G05)

Para que los estudiantes tengan éxito en el juego y obtengan una buena puntuación, es necesario que estos analicen todas las opciones que el juego ofrece para lograrlo. El hecho de obtener la mejor puntuación significa que... el alumno ha elegido la solución más correcta y por tanto, el niño ha aprendido. (G15)

Para finalizar esta dimensión, a la pregunta si se pueden considerar los juegos de simulación como una innovación educativa, tan solo en 3 entrevistas grupales se dilucida con que probablemente estén suficientemente extendida su práctica y se lleve realizando durante demasiado tiempo como para considerarse como una innovación. En el resto de los equipos, sí se opina que todavía puede considerarse un recurso educativo innovador, principalmente porque su utilización es algo excepcional y poco tradicional (14 referencias) y porque es una herramienta didáctica entretenida, dinámica y divertida (13). A continuación se transcriben dos fragmentos sobre estas dos últimas categorías comentadas.

Es una buena forma de aprender con herramientas nuevas, diferentes a las que se suelen utilizar y que los niños saben manejar muy bien y aunque siempre suelen oponerlas al estudio, aqui se demuestra como juegos y educación pueden ir de la mano. (G07)

En definitiva, los videojuegos son una propuesta muy innovadora que puede hacer que los alumnos tengan el objetivo de superar los retos jugando e inconscientemente vayan aprendiendo los contenidos que pretendan los docentes enseñar, dependiendo del juego que les propongan... y pueden aprender de manera divertida y dinámica. (G16)

7.2.2. Aprendizajes de los juegos y sus posibilidades de transferencia

En las entrevistas grupales se insistió a estos estudiantes de un grado de educación sobre cuáles son los principales aprendizajes que se pueden extraer tras interactuar con los juegos analizados. De manera mayoritaria (21 referencias, $77,8 \%$ de las entrevistas grupales) se volvió a mencionar que fundamentalmente se aprendía a tomar decisiones y a reflexionar sobre ellas. En segundo lugar, se consideró que pueden mejorar los conocimientos específicos y las prácticas en el ámbito de la evaluación (11). También se destaca que se pueden trabajar aspectos como la asunción de responsabilidades (6), la capacidad de ser críticos (4), a tener valores sociales (3) o a organizarse y planificarse (3). A continuación, ejemplos de las categorías temáticas más referenciadas en esta cuestión:

Se aprende a tomar decisiones, analizarlas, reflexionar sobre las mismas, a hacerte responsable de tus actos, admitiendo las consecuencias de los mismos. (G19) 
Con "Un día con Eva", por un lado, lo que más he aprendido es a saber cómo evaluar y en qué debo fijarme para hacerlo $y$, por otro lado, a analizar las decisiones cotidianas y rutinarias. (G27)

Para complementar la argumentación sobre los aprendizajes, otra de las preguntas del guion de las entrevistas grupales versaba sobre si las competencias trabajadas en las situaciones de los juegos de simulación se podían transferir a la vida real. En este interrogante, los equipos solo opinan que no en el caso de "EVONG: Evaluación en Acción" porque se centra en un ambiente laboral muy específico (3 referencias). Las categorías más referenciadas, del que se muestra posteriormente un fragmento de cada una, es que sí se pueden transferir los aprendizajes porque, en el caso de "Un día con Eva”, refleja el día a día en la universidad (14) y porque realmente estamos evaluando, reflexionando y tomando decisiones constantemente en nuestras vidas (8).

En el de Eva teníamos que corregir los trabajos de los compañeros, tomar decisiones acerca de lo que hacer a lo largo del día, ir a clase, etc. Ejemplos que se asemejan a nuestro día a día y a lo que tenemos que aprender para ser buenos docentes. (G14)

Estas observaciones se pueden llevar perfectamente a la vida real ya que hemos descubierto que sin darnos cuenta, estamos continuamente evaluando, organizando y tomando decisiones, y cada una de esas decisiones tiene unas repercusiones de las que somos responsables. (G08)

\subsubsection{Propuestas de mejora}

El último ámbito en el que se profundizó en las entrevistas grupales fue en cómo consideraban que se podrían mejorar los juegos. En esta ocasión, las ideas han sido tan abundantes y diversas que se ha incluido el sistema de categorías de análisis completo en el cuadro 5 .

Cuadro 5. Propuestas de mejora para los juegos analizados

\begin{tabular}{|c|c|c|}
\hline CATEgoría & SUBCATEGORÍA & FRECUENCIA \\
\hline \multirow{2}{*}{$\begin{array}{l}\text { Diseño } \\
\text { gráfico }\end{array}$} & Mejorando los gráficos & 11 \\
\hline & Haciendo más naturales los movimientos de los personajes & 4 \\
\hline \multirow{5}{*}{ Actividades } & Eliminando texto en las lecturas & 4 \\
\hline & Ampliando y diversificando el tipo de actividades & 4 \\
\hline & Ajustando su dificultad al público destinatario & 4 \\
\hline & Teniendo la opción de oír el texto & 2 \\
\hline & Incluyendo un cuestionario final para reflexionar & 1 \\
\hline \multirow{4}{*}{ Jugabilidad } & Teniendo más libertad y opciones disponibles & 10 \\
\hline & Pudiendo elegir entre varios personajes & 1 \\
\hline & Haciéndolo más dinámico & 1 \\
\hline & Pudiendo elegir la vista $\left(1^{\mathrm{a}}\right.$ o $3^{\mathrm{a}}$ persona $)$ & 1 \\
\hline Orientaciones & Mejorando las instrucciones para hacerlas más comprensibles & 8 \\
\hline \multirow{2}{*}{ Duración } & Disponiendo de mayor duración & 5 \\
\hline & Realizándolo de menor duración & 1 \\
\hline \multirow{2}{*}{$\begin{array}{l}\text { Música y } \\
\text { sonidos }\end{array}$} & Cambiando la música por una más relajante & 1 \\
\hline & Mejorando los sonidos & 1 \\
\hline
\end{tabular}

Fuente: Elaboración propia.

Como se puede apreciar, las opiniones más recurrentes giran en torno a mejorar el diseño gráfico (11) y disponer de más libertad y de más opciones para jugar (10), de ambas categorías se ofrece un ejemplo: 
En cuanto al diseño, se podrían mejorar los movimientos de los personajes, ya que a veces resultan poco naturales, además de los gráficos. (GO2)

Si pudiésemos mejorar algún aspecto éste sería ampliar las oportunidades de interacción en el juego ya que esta está muy limitada a las pocas opciones que puedes escoger. (G11)

\section{Discusión y conclusiones}

La utilización de videojuegos educativos es cada vez más común en Educación Superior debido a sus posibilidades formativas y a que contribuyen a crear prácticas que implican activamente al alumnado. En este artículo se ha presentado una investigación sobre las percepciones de los estudiantes sobre dos juegos de simulación donde se recrea la realidad para interactuar con ella, con los que se pretende desarrollar el aprendizaje y la alfabetización en el ámbito de la evaluación.

Se ha comprobado, relacionando los resultados con los objetivos propuestos en este estudio, que los jugadores han considerado que la toma decisiones es la principal habilidad que se puede desarrollar, siendo igualmente el aspecto positivo más importante detectado. La toma de decisiones activa, junto con la reflexión y revisión de las consecuencias que conllevan es, a su vez, un aspecto primordial para autorregular el aprendizaje (García-Jiménez, 2015) y un elemento imprescindible en la mecánica de cualquier juego de simulación. Igualmente, otro de los aspectos destacables para los participantes es que plantea una ambientación muy realista con situaciones cercanas, algo que facilita una mayor inmersión en la dinámica de los juegos que podría favorecer una generalización de las estrategias utilizadas (Poy-Castro, Mendaña-Cuervo y González, 2015).

En general, se perciben como unas herramientas más entretenidas y novedosas que otros recursos educativos, aunque algunos participantes se plantean si los videojuegos no tienen ya la suficiente implantación para que dejen de considerarse como una innovación. Igualmente, si esta motivación se traduce en aprendizaje real sobre la evaluación, es una circunstancia que muchos de los estudiantes asocian inexcusablemente a la existencia de orientaciones formativas adecuadas, más allá del propio juego, que permitan mayores cotas de reflexión y profundización sobre las competencias y contenidos a trabajar. Por lo tanto, sería importante concluir que en los diseños de estos juegos serios siempre debería ser prioritario fomentar una motivación intrínseca con la propia narrativa y consecución de objetivos del juego respecto a una extrínseca basada únicamente en las puntuaciones y el avance interno (Rojo y Dudu, 2017), al igual como contar con suficientes orientaciones y apoyos didácticos que consigan dotarlos de coherencia formativa y sentido educativo.

Respecto al diseño gráfico de los juegos, no existe una opinión homogénea. Se aprecia en las aportaciones de los jugadores que la estética de ambos recursos es suficientemente buena y, al menos, no es considerada como un obstáculo para su disfrute. No obstante, para los participantes que interaccionan continuamente con videojuegos comerciales, no les han resultado especialmente atractivos, un aspecto importante a considerar en la creación propia de juegos simulación por parte de los docentes; y es que no sería realista competir en términos visuales con los videojuegos comerciales, con gran presupuesto y grandes equipos para su desarrollo, los cuales, aunque con evidentes beneficios educativos (Lacasa, 2010), no tienen suficiente contextualización ni especificidad para abordar determinadas competencias formativas, sobre todo en Educación Superior. 
Por otra parte y continuando con los objetivos propuestos, se ha comprobado que existen diferencias significativas en las valoraciones realizadas al juego "Un día con Eva" con respecto a "EVONG: Evaluación en Acción". Se ha puesto de manifiesto que el primero de ellos se percibe de una manera más satisfactoria, principalmente por considerarse que es más sencillo e intuitivo de jugar. De hecho, se manifiesta que los principales aspectos negativos de "Un día con Eva" es que puede resultar excesivamente fácil y que tiene unas opciones muy limitadas, mientras que para "EVONG: Evaluación en Acción" no resultan demasiado motivadoras las actividades y su jugabilidad y orientaciones son mejorables. Por lo tanto, un enfoque de diseño del juego sin demasiado texto, más cercano a la realidad, con un número suficiente de opciones y cuyas tareas no resulten especialmente formales y academicistas es el preferido por los estudiantes que han participado en la investigación.

Finalizando con nuestros objetivos y respecto a las posibilidades de transferencia de los aprendizajes de los juegos, se insistió en que principalmente sería la toma decisiones y prácticas de evaluación lo que se podría extrapolar a su experiencia académica y personal, ya que se puede ser consciente que la evaluación es una realidad que nos acompaña constantemente en nuestro día a día.

Continúa siendo imprescindible seguir con la indagación en esta temática educativa, abordando la creación de nuevos juegos de distintas tipologías para comparar su impacto en el aprendizaje en el ámbito de la evaluación. Los videojuegos de estrategia podrían ser una buena opción para potenciar el significado de los criterios de evaluación y favorecer la retroalimentación inmediata ante las decisiones a tomar, algo que vendría a complementar las posibilidades en la alfabetización en la evaluación (Price et al., 2012). Igualmente, se podrían abordar investigaciones desde enfoques más abiertos donde no se propongan afirmaciones tan abarcadoras, pues es una de las limitaciones fundamentales que se han detectado en el presente estudio, ya que se ha podido sesgar la amplitud argumental sobre las consideraciones de los participantes.

En definitiva, y a pesar de las dificultades crecientes para diseñar y editar autónomamente videojuegos educativos que puedan estar a la altura de los argumentos y la estética de los comerciales, se puede continuar apreciando el valor otorgado por los estudiantes de Educación Superior a las estrategias relacionadas con la gamificación como un valioso complemento formativo en sus carreras. La cercanía e interacción con una realidad controlada facilita la inmersión en historias donde el alumno se siente protagonista y donde se pueden practicar habilidades útiles y necesarias como las relacionadas con la evaluación, unas habilidades que en la realidad servirá para propiciar su participación en procesos evaluativos en la Educación Superior dentro de enfoques compartidos y formativos (Hortigüela, Pérez-Pueyo y González-Calvo, 2019), para favorecer su aprendizaje autónomo e incluso para llegar a ser conscientes de la relevancia multidimensional de la evaluación y hasta qué punto esta puede llegar a afectar su vida (McArthur, 2019).

\section{Referencias}

Boud, D. (2006). Foreword. En C. Bryan y K. Clegg (Eds.), Innovative Higher Education (pp. 1719). Londres: Routledge. 
Boud, D. et al. (2010). Assessment 2020: Seven propositions for assessment reform in higher education. Sydney: Australian Learning and Teaching Council.

Boud, D. y Falchikov, N. (2006). Aligning assessment with long-term learning. Assessment Eా Evaluation in Higher Education, $31(4)$, 399-413. https://doi.org/10.1080/02602930600679050

Brown, S. (2015). Perspectivas internacionales sobre la práctica de la evaluación en Educación Superior. RELIEVE, 21(1), art4. https://doi.org/10.7203/relieve.21.1.6403

Buchinger, D. y da Silva, M. (2018). Guidelines for designing and using collaborative-competitive serious games. Computers $\&$ Education, 118, 133-149. https://doi.org/10.1016/j.compedu.2017.11.007

Chen, K. H. y Hoffman, J. (2017). Serious Play: Transforming Futures Thinking Through Gamebased Curriculum Design. Journal of Futures Studies, 22(2), 41-60. https://doi.org/10.6531/JFS.2017.22(2).A41

De Freitas, S. y Oliver, M. (2006). How can exploratory learning with games and simulations within the curriculum be most effectively evaluated? Computers $\mathcal{F}^{\circ}$ Education, 46(3), 249264. https://doi.org/10.1016/j.compedu.2005.11.007

García-Jimenez, E. (2015). La evaluación del aprendizaje: de la retroalimentación a la autorregulación. El papel de las tecnologías. RELIEVE, 21(2), art. M2. https://doi.org/ 10.7203/relieve.21.2.7546

Gómez-Ruiz, M. A., Rodríguez-Gómez, G. e Ibarra-Sáiz, M. S. (2013). Desarrollo de las competencias básicas de los estudiantes de Educación Superior mediante la e-Evaluación orientada al aprendizaje. RELIEVE, 19(1), art. 1.

https://doi.org/10.7203/relieve.19.1.2457

Gros-Salvat, B. (2009). Certezas e interrogantes acerca del uso de los videojuegos para el aprendizaje. Comunicación: Revista Internacional de Comunicación Audiovisual, Publicidad y Estudios Culturales, 7(1), 251-264.

Hattie, J. y Timperley, H. (2007). The power of feedback. Review of Educational Research, 77(1), 81-112. https://doi.org/10.3102/003465430298487

Hayward, L. (2015). Assessment is learning: the preposition vanishes. Assessment in Education: Principles, Policy \& Practice, 22(1), 27-43. https://doi.org/10.1080/0969594X.2014.984656

Hortigüela, D., Pérez-Pueyo, Á. y González-Calvo, G. (2019). Pero... ¿A qué nos referimos realmente con la evaluación formativa y compartida?: Confusiones habituales y reflexiones prácticas. Revista Iberoamericana de Evaluación Educativa, 12(1), 13-27.

https://doi.org/10.15366/riee2019.12.1.001

Ibarra-Sáiz, M. S. y Rodríguez-Gómez, G. (2017). Serious Games for Students' E-Assessment Literacy in Higher Education. En E. Cano y G. Ion (Eds.), Innovative Practices for Higher Education Assessment and Measurement (pp. 271-294). Derry, PE: IGI Global. https://doi.org/10.4018/978-1-5225-0531-0.ch014

Ibarra-Sáiz, M. S. y Rodríguez-Gómez, G. (2019). Evaluación como aprendizaje. En J. Paricio, A. Fernández e I. Fernández (Eds.), Cartografía de la buena docencia universitaria. Un marco para el desarrollo del profesorado basado en la investigación (pp. 175-196). Madrid: Narcea.

Lacasa, P. (Coord.). (2010). Videojuegos Comerciales y Aprendizaje Escolar. Análisis de las creencias del alumnado de Educación Secundaria Obligatoria. Madrid: Universidad de Alcalá y Electronic Arts España.

McArthur, J. (2019). La evaluación: una cuestión de justicia social. Perspectiva crítica y prácticas adecuadas. Madrid: Narcea. 
Miles, M. B. y Huberman, A. M. (1994). Qualitative data analysis. A new sourcebook of methods. Beverly Hills, CA: Sage Publications.

Nadolski, R. J., Hummel, H. G., van den Brink, H. J., Hoefakker, R., Slotmater, A., Kurvers, H. J. y Storm, J. (2008). EMERGO: A Methodology and Toolkit for Developing Serious Games in Higher Education. Simulation \& Gaming, 39(3), 338-352. https://doi.org/10.1177/1046878108319278

Paradas-Castro, Á., Raposo-Rivas, M. y Martínez-Figueira, M. E. (2018). ¿Mejorar la atención con videojuegos? un estudio de caso. Revista Española de Orientación y Psicopedagogía, 29(3), 94-109. https://doi.org/10.5944/reop.vol.29.num.3.2018.23323

Penuel, W. R. y Shepard, L. A. (2016). Social models of learning and assessment. En A. A. Rupp y J. P. Leighton (Eds.), The handbook of cognition and assessment: Frameworks, methodologies, and applications (pp. 146-173). Hoboken, NJ: John Wiley \& Sons.

Pérez-Colado, I. J., Pérez-Colado, V. M., Martínez-Ortiz, I., Freire-Morán, M. y FernándezManjón, B. (2017). uAdventure: The eAdventure reboot - Combining the experience of commercial gaming tools and tailored educational tools. Proceedings of the IEEE Engineering Education Conference. Atenas: EDUCON. https://doi.org/10.1109/EDUCON.2017.7943087

Poy-Castro, R. Mendaña-Cuervo, C. y González, B. (2015). Diseño y evaluación de un juego serio para la formación de estudiantes universitarios en habilidades de trabajo en equipo. Revista lbérica de Sistemas y Tecnologías de Información, E3, 71-83. https://doi.org/10.17013/risti.e3.71-83

Price, M., Rust, C., O’Donovan, B., Handley, K. y Bryant, R. (2012). Assessment Literacy. The Foundation for Improving Student Learning. Oxford: Oxford Brookes University.

Quesada-Serra, V., García-Jiménez, E. y Gómez-Ruiz, M. A. (2017). Student participation in assessment processes: A way forward. En E. Cano y G. Ion (Eds.), Innovative practices for higher education assessment and measurement (pp. 228-249). Derry, PE: IGI Global. https://doi.org/10.4018/978-1-5225-0531-0.ch012

Rodríguez-Gómez, G. y Gómez-Ruiz, M. A. (2010). Análisis de contenido y textual de datos cualitativos. En S. Nieto Martín (Ed.), Principios, métodos y técnicas esenciales para la investigación educativa (pp. 447-469). Madrid: Dikynson.

Rodríguez-Gómez, G. e Ibarra-Sáiz, M. S. (noviembre, 2014). Desarrollo de la competencia evaluadora en estudiantes universitarios a través de juegos de simulación. Comunicación presentada en el Congreso Iberoamericano de Ciencia, Tecnología, Innovación y Educación, Organización de Estados Iberoamericanos, Buenos Aires.

Rojo, T. y Dudu, S. (2017). Los “juegos serios" como instrumento de empoderamiento y aprendizaje socio-laboral inclusivo. Revista Fuentes, 19(2), 95-109.

https://doi.org/10.12795/revistafuentes.2017.19.2.07

Santos, A. F., Alloza, S. y Escribano, F. (2018). Manual para educadores: Relación entre géneros de videojuegos y soft skills. Recuperado de

https://gecon.es/wp-content/uploads/2018/04/gecon.es-

Genero_videojuegos_soft_skills.pdf

Sedeño, A. (2010). Videojuegos como dispositivos culturales: las competencias espaciales en educación. Comunicar: Revista Científica de Comunicación y Educación, 34, 183-189. https://doi.org/10.3916/C34-2010-03-18

Serrano-Laguna, E., Marchiori, E. J., Del Blanco, A., Torrente, J. y Fernández-Manjón, B. (2012). A framework to improve evaluation in educational games. Proceedings of the IEEE 
Engineering Education Conference. Marrakesh: EDUCON. https://doi.org/10.1109/EDUCON.2012.6201154

Smith C. D., Worsfold, K., Davies, L., Fisher, R. y McPhail, R. (2013). Assessment literacy and student learning: the case for explicitly developing students 'assessment literacy'. Assessment Eं Evaluation in Higher Education, 38(1), 44-60. https://doi.org/10.1080/02602938.2011.598636

Squire, K. (2003). Video games in education. International Journal of Intelligent Simulations and Gaming, 2(1), 49-62.

Teixes, F. (2015). Gamificacion: Motivar jugando. Barcelona: Editorial UOC.

Urquidi-Martín, A. C. (2014). Juegos de simulación como estrategia didáctica en la universidad. En J. E. Gonzálvez-Vallés (Coord.), Nuevas tendencias en innovación educativa superior (pp. 431-450). Madrid: Asociación Cultural y Científica Iberoamericana.

Zamora-Enciso, R. (2010). Competencias socio-emocionales: su desarrollo a través del juego y la simulación. Madrid: Lulu.

\section{Agradecimientos}

El juegos de simulación "Un día con Eva" fue diseñado en el contexto del Proyecto DevalSimWeb: Desarrollo de competencias profesionales a través de la evaluación participativa y la simulación utilizando herramientas web $\left(\mathrm{N}^{\circ}\right.$ DCIALA/19.09.01/11/21526/264-773/ALFAIII(2011)-10). Financiado por la Comisión Europea.

El juego de simulación "EVONG: Evaluación en Acción" fue diseñado en el contexto del Proyecto DevalS: Desarrollo de la e-Evaluación sostenible. Mejora de la competencia evaluadora de los estudiantes universitarios mediante simulaciones virtuales. Financiado por el Ministerio de Economía y Competitividad. Convocatoria del Plan Nacional $\mathrm{I}+\mathrm{D}+\mathrm{i}$. Referencia: EDU2012-31804.

\section{Breve $\mathrm{Cv}$ de los autores}

\section{Miguel Ángel Gómez Ruiz}

Doctor en Psicología, Educación y Desarrollo, actualmente es Profesor Ayudante Doctor en el Área de Didáctica y Organización Escolar del Departamento de Didáctica de la Universidad de Cádiz. Miembro del Grupo de Investigación EVALfor (Evaluación en Contextos Formativos - SEJ-509). Investigador de la Cátedra UNESCO en Evaluación, Innovación y Excelencia de la Universidad de Cádiz. Sus líneas de investigación sobre evaluación se centran en la evaluación orientada al aprendizaje, la utilización de recursos y aplicaciones tecnológicas en evaluación, la participación en procesos evaluativos y la evaluación dialógica y compartida. Sobre estas temáticas ha realizado diversas publicaciones $\mathrm{y}$ ha participado en numerosos proyectos de investigación financiados en convocatorias públicas. ORCID ID: https://orcid.org/o000-0003-2418-9525. Email: miguel.gomez@uca.es 


\section{María Soledad Ibarra Sáiz}

Profesora Titular en el Área de Métodos de Investigación y Diagnóstico en Educación de la Universidad de Cádiz. Doctora y licenciada en Ciencias de la Educación y licenciada en Psicología. Directora de la Cátedra UNESCO Evaluación, Innovación y Excelencia en Educación desde 2018. Directora del Grupo de Investigación EVALfor SEJ 509-Evaluación en contextos formativos desde 2004. Directora de Innovación, Convergencia y Formación de la Universidad de Cádiz (2007-2011). Especialista em evaluación en la Educación Superior, siendo las principales líneas: la evaluación como aprendizaje y empoderamiento y las tecnologías que favorecen la evaluación, desarrolladas a través de diferentes proyectos de investigación dirigidos y difundidas en numerosos artículos, capítulos de libro, libros y congresos internacionales. ORCID ID: https://orcid.org/0000-0003-4513-702X. Email: marisol.ibarra@uca.es

\section{Gregorio Rodríguez Gómez}

Doctor en Ciencias de la Educación. Catedrático de Universidad de Métodos de Investigación en Educación de la Universidad de Cádiz. Investigador del Grupo de Investigación EVALfor SEJ 509 - Evaluación en contextos formativos y coordinador del área de Estudios e investigaciones de la Cátedra UNESCO Evaluación, Innovación y Excelencia en Educación. Miembro de la Comisión de Evaluación del Programa AUDIT (2008-2011) y evaluador del Programa de Evaluación Institucional (convocatorias 2005, 2006, 2007). Es experto externo para la evaluación del Criterio 3 "Sistema de Garantía Interno de Calidad" en los programas ACREDITA y MONITOR de la ANECA. Ha sido Presidente de la Asociación Interuniversitaria de Investigación Pedagógica (AIDIPE) y en la actualidad es Presidente de la Red Estatal de Docencia Universitaria (REDU). ORCID ID: https://orcid.org/o000-0001-9337-1270. Email: gregorio.rodriguez@uca.es 